\title{
Aplicación del Inventario de Trastornos de la Alimentación (EDI) en Estudiantes de Salud
}

\section{Application of Inventory Method (EDI) in Health Students Who Develop Eating Disorders}

Recibido 1 Jul. 2013/Enviado para Modificación 30 Oct. 2013/Aceptado 18 Nov. 2013

\author{
Anderson Díaz Pérez ${ }^{1}$ \\ Corporación Universitaria Rafael Núñez \\ Universidad Popular del Cesar \\ Angélica Tortello Martínez ${ }^{2}$ \\ Universidad Popular del Cesar \\ Arley Vega Ochoa ${ }^{3}$ \\ Universidad Popular del Cesar \\ Moraima del Toro Rubio ${ }^{4}$ \\ Corporación Universitaria Rafael Núñez
}

\section{RESUMEN}

Introducción: En los últimos 30 años, la anorexia y la bulimia nerviosa han adquirido especial relevancia como proceso patológico en las mujeres adolescentes y en adultos jóvenes sin exclusión socioeconómica. Objetivo: Identificar las características psicológicas y comportamentales relacionadas con trastornos alimenticios en universitarias de la Facultad de Ciencias de la Salud en Cartagena de Indias. Materiales y Métodos: Se realizó un estudio descriptivo de corte transversal. El muestreo fue de 150 mujeres de programas de pregrado de la Facultad De Salud de la Corporación Universitaria Rafael Núñez de Cartagena de Indias. Se utilizó la escala de Inventario de Desórdenes Alimentarios (EDI) que consta de 8 subescalas: impulso a la delgadez, sintomatología bulímica, insatisfacción corporal, inefectividad y baja autoestima, perfeccionismo, desconfianza interpersonal, conciencia interoceptiva y miedo a afrontar la vida adulta. Resultados: En odontología, se presentó una tendencia por el impulso hacia la delgadez en un 14.4\% de las participantes, en bacteriología, se dio sintomatología bulímica en 5.8\%, también en odontología se presentó un rasgo de insatisfacción corporal en un $15 \%$, y en medicina, inefectividad y baja autoestima en un 9\%. Además, en bacteriología, se evidenció el rasgo de perfeccionismo en un $19.1 \%$; y en medicina, el miedo a afrontar la vida adulta en un $23.9 \%$. Conclusión: el temor de enfrentar la vida adulta era la característica más relevante entre los estudiantes.

Palabras Clave: Trastornos de la Alimentación, Adolescentes, Anorexia, Bulimia Nerviosa (Fuente: DeCS).

\section{ABSTRACT}

Introduction: Anorexia and bulimia nervosa have gained particular relevance as pathological process in adolescent women and in young adults without socioeconomic exclusion, in the last 30 years. Objective: To identify the psychological and behavioral features associated with students who develop eating disorders in the faculty of Health Sciences in universities from Cartagena de Indias. Materials and Methods: Cross sectional descriptive study was applied. The sampling was of 150 women from undergraduate programs of the Faculty of Health at Rafael Nuñez University from Cartagena de Indias. A scale for eating disorders inventory method (EDI) was applied. This one, consists of 8 subscales: momentum to thinness, bulimic symptomatology, body dissatisfaction, ineffectiveness and low self-esteem, perfectionism, interpersonal distrust and fear of confronting the adult life Results: In odontology, a tendency to the momentum to thinness in $14.4 \%$ of the participants was evident, and a feature of corporal dissatisfaction appeared in $15 \%$ as well. In bacteriology, bulimic symptomatology was shown in $5.8 \%$ and the perfectionism feature was shown in $19.1 \%$. In medicine, ineffectiveness and low self-esteem in 9\%; in both bacteriology and medicine, the fear of confronting adult life was the most relevant characteristic among students. Conclusion: the fear of confronting adult life was the most relevant characteristic among students.

Keywords: Eating Disorders, Adolescents, Anorexia, Bulimia Nervosa (Source: MeSH, NLM).

1 Instrumentador Quirúrgico. Magister en Ciencias Básicas Biomédicas. Doctorando en Bioética. Coordinador de investigación del programa de Enfermería de la Corporación Universitaria Rafael Núñez. Docente e investigador de la Universidad Popular del Cesar. Correo electrónico: ander2711@gmail.com

2 Enfermera. Especialista en Salud Familiar y Maestrante en Educación. Docente e Investigadora del programa de Enfermería. Universidad Popular del Cesar

3 Instrumentadora Quirúrgica. Especialista en Gestión Educativa. Magíster en Salud Ocupacional. Docente e Investigadora del programa de Instrumentación Quirúrgica. Universidad Popular del Cesar.

4 Enfermera. Especialista en Salud Familiar y Magíster en Educación. Docente investigadora. Corporación Universitaria Rafael Núñez 


\section{Introducción}

Los Trastornos del Comportamiento Alimentario (TCA) anorexia y bulimia nerviosa, junto a cuadros afines o no especificados, han alcanzado en los últimos 30 años una especial relevancia, constituyéndose en una enfermedad identificada principalmente en la población femenina (1), pues, sólo del $5 \%$ a $10 \%$ de los casos involucran hombres (2). En nuestra sociedad, el afán de adelgazar se ha convertido en una auténtica obsesión, de modo que el índice de mujeres que se sienten desgraciadas a causa de su físico, y en especial de su exceso de peso, sea este real o imaginario, aumenta día a día (3).

Muchos estudios muestran datos sobre la influencia de los genes, la asociación de neurocircuitos, la bioquímica y la fisiología, así como otros factores de riesgo psicosociales, que predisponen al desarrollo de trastornos de la alimentación (4); sin embargo, esto no excluye la necesidad de diseños y ampliación de modelos preventivos y de tratamiento, teniendo presente los riesgos biopsicosociales, como por ejemplo: la adversidad familiar o factores ambientales, que actúan como un fenómeno multi-causal que puede alterar la expresión génica a través de procesos epigenéticos y propiciar la manifestación del trastorno (5).

Las personas que padecen algún tipo de trastorno alimenticio dan una especial importancia a ciertos alimentos alrededor de los cuales giran todos sus pensamientos, sentimientos, sueños, ideales y problemas, y se hacen dependientes de esas ideas, con lo cual tales alimentos se convierten en el eje principal de su vida. Se sabe que una dieta estricta constituye el factor de riesgo más importante para desarrollar estos trastornos, ya que regularmente las mujeres que se someten a este tipo de dietas, aumentan dieciocho veces sus probabilidades de desarrollar un trastorno alimenticio (6).

En el campo de la salud mental, la anorexia nerviosa es por sí sola la enfermedad de más alta mortalidad en el mundo, hasta el punto que cobra la vida de entre 5 a $20 \%$ de las personas que la padecen. Y, contrario a lo que se piensa, la anorexia y la bulimia no son enfermedades propias de las clases socioeconómicas altas. Por otra parte, los trastornos de la alimentación nunca se presentan solos, y más del $90 \%$ de las personas que los padecen presentan además depresión y/o trastornos de ansiedad (7).
Los estudiantes de Ciencias de la Salud se exponen continuamente a factores estresantes por circunstancias tales como: asignaturas que suman un alto número de créditos, poco tiempo libre, abuso del alcohol, entre otros. Todo lo cual conforma al grupo de factores desencadenantes para la manifestación de TCA (8). En este contexto, los autores de la presente investigación se propusieron conocer los rasgos psicológicos y comportamentales de la población universitaria, en concordancia con el principal factor de riesgo, que es la percepción de la imagen corporal (9).

Los desórdenes alimentarios se dividen en tres categorías diagnósticas: anorexia nerviosa (AN), bulimia nerviosa (BN) y los trastornos de la conducta alimentaria no especificados (TCA-NOE). Los principales criterios diagnósticos para $\mathrm{AN}$ son la sobrevaloración de la figura (imagen corporal) y el peso, mantener un peso muy bajo (IMC $\leq 17.5 \mathrm{~kg} / \mathrm{m} 2$ ), y además amenorrea en mujeres fértiles que no usan anticonceptivos, aunque esto último no siempre se presenta. En la BN, también aparece la sobrevaloración de la figura y del peso, además de recurrentes episodios de atracones, extremo control de peso (dietas restrictivas, inducción de vómito frecuente, o el uso de laxantes). Los TCA-NOE se definen por la presencia incompleta de los criterios diagnósticos para $\mathrm{AN}$ y $\mathrm{BN}$ en sus diferentes combinaciones (ausencia de uno o de los dos) (7, 10, 11). Estos desórdenes alimentarios están definidos en el Diagnostic and Statistical Manual of Mental Disorders (DSM IV) $(12,13)$. Los trastornos alimentarios ocurren en $1 \%$ y $4 \%$ de los adolescentes y mujeres adultas jóvenes, predominantemente de raza blanca, y pertenecientes a la clase media y media-alta (7).

Como se ha dicho, el componente genético y los factores medioambientales son importantes para la aparición de los TCA (14). Además, estos trastornos se han relacionado con un alto riesgo de morbilidad junto a otros desórdenes psiquiátricos (particularmente el desorden obsesivo-compulsivo) y a un alto riesgo de muerte a temprana edad por suicidio o como consecuencia de alteraciones fisiológicas debidas a la mala alimentación, es decir, a la etiología multifactorial $(14,15)$.

En el ámbito universitario, se presentan varios factores de riesgo para sufrir TCA como son: las crisis depresivas, el estrés, la ansiedad, etc., con el agravante de que la población estudiada está inmersa en el rango

Cienc. innov. salud. Diciembre 2013; 1 (2):101 - 107. Universidad Simón Bolívar (Col). ISSN: 2344-8636 http://portal.unisimonbolivar.edu.co:82/rdigital/innovacionsalud 
de edad de riesgo para algún tipo de TCA: (12 a 25 años) (16). Existen pocos estudios epidemiológicos del riesgo de padecer desórdenes alimentarios en adolescentes y adultos jóvenes en países desarrollados y en América Latina $(17,18)$; por lo cual se crea la necesidad de desarrollar estrategias de filtro (tamización) y de diagnóstico temprano, tanto en las escuelas como en universidades del país.

El objetivo de nuestro trabajo es comparar, entonces, las características psicológicas y comportamentales relacionadas con los trastornos de la conducta alimentaria (Bulimia Nerviosa o Anorexia Nerviosa), a los que están bastante propensas las estudiantes de la Facultad de Ciencias de la Salud de una Universidad Privada de Cartagena de Indias, a las que se aplicó la escala de EDI como principal estrategia de tamización y diagnóstico temprano.

\section{Materiales y Métodos}

El tipo de estudio utilizado fue el descriptivo, comparativo de corte Transversal. El muestreo se realizó al azar, escogiéndose 150 mujeres de diferentes edades de los programas de Bacteriología, Enfermería, Instrumentación Quirúrgica, Medicina y Odontología de la Facultad de Ciencias de la Salud de la Corporación Universitaria Rafael Núñez de Cartagena, Colombia. Para determinar la posible prevalencia de los TCA, se consideraron 30 mujeres de cada programa, entre el primero y el último semestre del 2011 al 2012, a quienes se aplicó el Inventario de Trastornos de la Alimentación-EDI para anorexia nerviosa, bulimia nerviosa o trastornos de la alimentación no especificados.

Vale la pena indicar algunas limitaciones de dicho inventario, como son su orientación exclusiva a mujeres, y su excesiva longitud (64 ítems).

El EDI se aplicó teniendo en cuenta su clasificación en 8 subescalas, con posibilidades de respuestas, seis de ellas con opciones de «siempre» a «nunca», y tres, con puntuación de 1 a 3 . La tabulación de los datos se realizó en el programa de Excel 2007, para su posterior análisis en el programa IBM SPSS Statistics 20.0 .

La puntuación total de este cuestionario puede oscilar entre 0 y 192. Desde el punto de vista clínico, posee mayor relevancia el valor cuantitativo de cada una de las ocho subescalas que la puntuación total. Las subescalas están divididas de la siguiente manera: impulso a la delgadez (7 ítems), sintomatología bulímica (7 ítems), insatisfacción corporal (9 ítems), inefectividad y baja autoestima (10 ítems), perfeccionismo (6 ítems), desconfianza interpersonal (7 ítems), conciencia interoceptiva (10 ítems), miedo a madurar (8 ítems).

La información fue recolectada de acuerdo con la naturaleza y grado de medición de cada variable, a través de un instrumento objetivo aplicado por el grupo investigador de estudiantes de enfermería de VIII semestre de la Corporación Universitaria Rafael Núñez. El instrumento permitió realizar una preselección de las estudiantes que presentaban algún tipo de conducta considerada de riesgo con respecto a un TCA. Algunas preguntas debieron ser validadas para una mejor compresión del estudiante, pero sin cambiar el fundamento de la pregunta original.

\section{Componente ético}

La aplicación de los instrumentos se realizó previa firma del consentimiento informado (CI) aprobado por el comité de investigación de la facultad de Ciencias de la Salud. El CI se diseñó teniendo presente la declaración de Helsinki y la resolución 0008430/93, considerándolo sin riesgo. El consentimiento se socializó de manera verbal y escrita a cada una de las participantes, a través de estas estrategias se les explicó en qué consistía la investigación y cómo se darían a conocer los resultados, aclarando que podían negarse a participar en cualquier momento del estudio.

La información fue analizada según la naturaleza y grado de medición de cada variable, cuestión que se resume en dos pasos: La tabulación y las gráficas de barras y de pastel, que se realizaron con el programa Excel 2007. Para la realización de las tablas de frecuencias, porcentajes y medidas de tendencia central, etc., se utilizó el programa Statgraphics Centurión XVII con un nivel de confianza del 95\%.

\section{Resultados}

El grupo de estudio fue conformado por 150 estudiantes, que cursaban del primero al último semestre de los programas de Medicina, Enfermería, Instrumentación Quirúrgica, Bacteriología y Odontología de la Corporación Universitaria Rafael Núñez, que contaban con una media de edad de 20

Cienc. innov. salud. Diciembre 2013; 1 (2):101 - 107. Universidad Simón Bolívar (Col). ISSN: 2344-8636 
años, aunque la mayoría tenía 19, en tanto que la edad mínima fue de 16 años y la máxima de 35 (Tablas 1 y 2).

Tabla 1. Edad del total de estudiantes de la Facultad de Ciencias de la Salud

\begin{tabular}{ll}
\hline Medida Estadística & Valor \\
\hline Media & 20,31 \\
Mediana & 20 \\
Moda & 19 \\
Desv. Típ. & 2,62 \\
Varianza & 6,87 \\
Rango & 19 \\
Mínimo & 16 \\
Máximo & 35 \\
Total & 150 \\
\hline
\end{tabular}

Entre los factores psicológicos y comportamentales considerados de riesgo para la presencia de TCA medidos con el instrumento EDI, se encontró que en el programa de Odontología hay una tendencia por el impulso a la delgadez en un $14.4 \%$; en el programa de Instrumentación Quirúrgica, en un $13.4 \%$ y en el de Enfermería, en un $11.5 \%$ (Tabla 3).

El programa de Medicina presentó la sintomatología de bulimia en un $3.1 \%$, y el de Bacteriología en un $5.8 \%$ (Tabla 3).

Con relación al rasgo de insatisfacción corporal, el programa de Odontología obtuvo un $15 \%$, seguido de Medicina (14.1\%) y en menor proporción, de Bacteriología, con un $10.6 \%$. La inefectividad y baja autoestima se presentó en un porcentaje del $9 \%$ en el programa de Medicina. A este le siguió el de Enfermería en un $8.5 \%$ y, por último, Instrumentación Quirúrgica en un 5.8\% (Tabla 3).

Tabla 2. Edades de los estudiantes por programa académico

\begin{tabular}{cccccc}
\hline Edad en años & $\begin{array}{c}\text { Bacteriología } \\
\mathbf{n}(\boldsymbol{\%})\end{array}$ & $\begin{array}{c}\text { Enfermería } \\
\mathbf{n}(\boldsymbol{\%})\end{array}$ & $\begin{array}{c}\text { Instrumentación Quirúrgica } \\
\mathbf{n}(\boldsymbol{\%})\end{array}$ & $\begin{array}{c}\text { Medicina } \\
\mathbf{n}(\%)\end{array}$ & $\begin{array}{c}\text { Odontología } \\
\mathbf{n}(\%)\end{array}$ \\
\hline 16 & $0(0)$ & $0(0)$ & $1(3.3)$ & $0(0)$ & $0(0)$ \\
17 & $1(3.3)$ & $6(20)$ & $6(20)$ & $0(0)$ & $0(0)$ \\
18 & $5(16.7)$ & $5(16.7)$ & $5(16.7)$ & $2(6.7)$ & $5(16.7)$ \\
19 & $6(20)$ & $5(16.7)$ & $7(23.3)$ & $9(30)$ & $3(10)$ \\
20 & $5(16.7)$ & $5(16.7)$ & $7(23.3)$ & $6(20)$ & $4(13.3)$ \\
21 & $4(13.3)$ & $2(6.7)$ & $1(3.3)$ & $5(16.7)$ & $6(20)$ \\
22 & $0(0)$ & $1(3.3)$ & $2(6.7)$ & $6(20)$ & $4(13.3)$ \\
23 & $4(13.3)$ & $2(6.7)$ & $0(0)$ & $0(0)$ & $5(16.7)$ \\
24 & $3(10)$ & $1(3.3)$ & $0(0)$ & $0(0)$ & $2(6.7)$ \\
25 & $1(3.3)$ & $2(6.7)$ & $0(0)$ & $1(3.3)$ & $0(0)$ \\
26 & $0(0)$ & $0(0)$ & $0(0)$ & $1(3.3)$ & $1(3.3)$ \\
27 & $2(3.3)$ & $0(0)$ & $0(0)$ & $0(0)$ & $0(0)$ \\
29 & $0(0)$ & $0(0)$ & $0(0)$ & $0(0)$ & $0(0)$ \\
35 & $0(0)$ & $1(3.3)$ & $1(3.3)$ & $0(0)$ & $0(0)$ \\
Total & $30(100)$ & $30(100)$ & $30(100)$ & $30(100)$ & $30(100)$ \\
\hline
\end{tabular}

n: número de estudiantes. (\%): Porcentaje de estudiantes

Entre los factores psicológicos y comportamentales considerados de riesgo para la presencia de TCA medidos con el instrumento EDI, se encontró que en el programa de Odontología hay una tendencia por el impulso a la delgadez en un $14.4 \%$; en el programa de Instrumentación Quirúrgica, en un $13.4 \%$ y en el de Enfermería, en un $11.5 \%$ (Tabla 3).
El programa de Medicina presentó la sintomatología de bulimia en un $3.1 \%$, y el de Bacteriología en un $5.8 \%$ (Tabla 3).

Con relación al rasgo de insatisfacción corporal, el programa de Odontología obtuvo un $15 \%$, seguido de Medicina (14.1\%) y en menor proporción, de Bacteriología, con un $10.6 \%$. La inefectividad y baja autoestima se presentó en un porcentaje del $9 \%$ en el 
programa de Medicina. A este le siguió el de Enfermería en un $8.5 \%$ y, por último, Instrumentación Quirúrgica en un 5.8\% (Tabla 3).

El perfeccionismo apareció con $19.1 \%$ en el programa de Bacteriología, un $18.4 \%$ en el programa de Instrumentación Quirúrgica y, por último, con un $12.7 \%$, en el programa de Medicina (Tabla 3). En lo correspondiente a la desconfianza interpersonal, el programa de Medicina presentó un 13.2\%, seguido de Enfermería, en un $12.4 \%$. Y en cuanto a la conciencia interoceptiva, el programa de Bacteriología alcanzó un porcentaje del 15.6\%, Instrumentación Quirúrgica, un $14.3 \%$, y el que menor conciencia interoceptiva tuvo fue el programa de Odontología, que marcó un 10.5\% (Tabla 3). El rasgo de miedo a madurar alcanzó el mayor puntaje en el programa de Medicina, con 23.9\%, seguido del programa de Instrumentación Quirúrgica, con $22.5 \%$, y en un menor porcentaje el programa de Bacteriología, con 19.4\% (Tabla 3).

Tabla 2. Subescalas para valorar el riesgo de los estudiantes de los programas a algún tipo de trastornos alimenticios

\begin{tabular}{lccccc}
\hline $\begin{array}{c}\text { RASGOS PSICOLÓGICOS } \\
\text { Y COMPORTAMENTALES } \\
\text { DE LOS ESTUDIANTES }\end{array}$ & $\begin{array}{c}\text { Bacteriología } \\
\mathbf{n}(\boldsymbol{\%})\end{array}$ & $\begin{array}{c}\text { Enfermerí } \\
\mathbf{a} \\
\mathbf{n}(\boldsymbol{\%})\end{array}$ & $\begin{array}{c}\text { Instrumentació } \\
\mathbf{n} \text { Quirúrgica } \\
\mathbf{n}(\boldsymbol{\%})\end{array}$ & $\begin{array}{c}\text { Medicina } \\
\mathbf{n}(\boldsymbol{\%})\end{array}$ & $\begin{array}{c}\text { Odontología } \\
\mathbf{n}(\boldsymbol{\%})\end{array}$ \\
\hline Impulso a la delgadez & $146(12)$ & $147(11.5)$ & $163(13.4)$ & $133(13)$ & $188(14.4)$ \\
Sintomatología bulímica & $71(5,8)$ & $55(4.3)$ & $57(4.7)$ & $32(3.1)$ & $55(4.2)$ \\
Insatisfacción corporal & $129(10,6)$ & $154(12.1)$ & $142(11.7)$ & $144(14.1)$ & $197(15)$ \\
Inefectividad y baja autoestima & $79(6.5)$ & $108(8.5)$ & $71(5.8)$ & $92(9)$ & $103(7.9)$ \\
Perfeccionismo & $232(19.1)$ & $210(16.4)$ & $223(18.4)$ & $130(12.7)$ & $212(16.2)$ \\
Desconfianza interpersonal & $132(10.9)$ & $159(12.4)$ & $111(9.1)$ & $135(13.2)$ & $155(11.8)$ \\
Conciencia interoceptiva & $189(15.6)$ & $174(13.6)$ & $174(14.3)$ & $113(11)$ & $138(10.5)$ \\
Miedo a madurar & $236(19.4)$ & $271(21.2)$ & $273(22.5)$ & $245(23.9)$ & $261(19.9)$ \\
\hline
\end{tabular}

n: número de estudiantes. (\%): Porcentaje de estudiantes

\section{Discusión}

Los trastornos alimenticios pertenecen a la clasificación de enfermedades psicosomáticas constituyen uno de los principales problemas de salud más frecuentes en los adolescentes, asociados incluso a otros tipos de patologías, como demencia y suicidio. El porcentaje de este último se encuentra entre el 1.8 al $7.3 \%$ asociado con algún tipo de TCA $(19,20)$.

La bulimia y la anorexia nerviosa son los trastornos más frecuentes a nivel psiquiátrico. Lo anterior se demostró con el tamizaje realizado en la presente investigación, al identificar las características psicológicas y comportamentales como enfermedades psicosomáticas que figuran entre los problemas de salud crónicos más frecuentes en los adolescentes, sobre todo entre edades de 10 a 24 como lo señalan otros autores $(2,21)$, que nuestro estudio corrobora entre edades de 16 a 20.
La detección temprana o tamizaje podría disminuir la letalidad de estos trastornos, que se asocian con el suicidio o con otros tipos de trastornos psiquiátricos, aunque la anorexia y bulimia nerviosa son las más detectadas. La presente investigación indicó, en el cuadro de características psicológicas y comportamentales, que la imagen corporal y la baja autoestima por la influencia sociocultural median de alguna manera el comportamiento psicológico de los adolescentes y jóvenes (3). En conjunto, estos rasgos predisponen de manera individual a cada estudiante a un tipo de trastorno psicosomático específico que se relaciona con los trastornos alimenticios (22).

Dados los resultados descritos, entre las principales recomendaciones de nuestro estudio aparece la necesidad de implementar estudios longitudinales de tipo transcultural, para plantear estrategias de seguimiento con criterios específicos y establecer el diagnóstico del tipo de trastorno alimenticio o psicosomático al cual están predispuestos los estudiantes (23) 
Las diferentes subescalas del EDI mostraron los rasgos psicológicos y comportamentales comunes a la anorexia nerviosa y bulimia nerviosa en los estudiantes de los 5 programas. El miedo a madurar fue la variable más alta en todos los análisis realizados, seguida por el perfeccionismo. La investigación sugirió, además, que es necesario identificar los factores de riesgo que influyen en las mujeres para que padezcan este tipo de trastornos de conducta alimentaria, entre los que se cuentan: factores sociales, ambientales y personales como la insatisfacción o la obsesión, los cuales se cuentan entre los rasgos psicológicos mostrados en la Tabla 2.

En el grupo de estudiantes, se percibe un riesgo hacia los trastornos alimentarios como la ingesta y el impulso a la delgadez que aparecen como uno de los síntomas más predisponentes a la bulimia y anorexia nerviosa, debido a los dramáticos esfuerzos por controlar el peso y la silueta, además de los diferentes trastornos condicionados por la falta de autoestima: insatisfacción corporal, el perfeccionismo como elemento de aceptación social, desconfianza interpersonal y la falta de reconocimiento de sus propias emociones y sensaciones (conciencia interoceptiva). Todo ello sumado al miedo a madurar o a adquirir responsabilidades, sobre todo entre las edades de 16 a 20 años, cuando están por culminar la profesión (Tabla 1).

En síntesis, en el presente estudio, se encontró que los estudiantes corren un alto riesgo de presentar la anorexia y la bulimia nerviosa, debido a los rasgos psicológicos y comportamentales encontrados. Esto conlleva a proponer o diseñar un programa de tamizaje e intervención en todas las escuelas y universidades del país. También se sugiere ampliar el estudio a otros programas de diferentes facultades, tomando en cuenta la intensidad horaria de las clases, falta de tiempo libre, exigencia académica a la hora de los exámenes, exigencias propias de la institución, factores familiares, económicos, socio-culturales, lo cual hizo falta determinar en la presente investigación, debido al principal propósito de la misma.

\section{Agradecimientos}

A los estudiantes de Enfermería Juan Guillermo Barrantes Vera, Marco Antonio Conrado Patiño, Carlos Arturo Díaz Suazman y Andrés Mauricio Molinares Arismendi, por su colaboración en la aplicación del instrumento a los estudiantes de pregrado. También a los estudiantes, padres, docentes, personal y administrativo de cada una de las dependencias de la universidad, que participaron de manera directa o indirecta del presente estudio. A la Corporación Universitaria Rafael Núñez, por permitir la realización del presente estudio en sus instalaciones y brindarnos el apoyo de los docentes al momento de la aplicación de los instrumentos a los estudiantes de la Facultad de Ciencias de la Salud.

\section{Referencias}

1. Cenci M, Peres KG, Vasconcelos FA. Prevalência de comportamento bulímico e fatores associados em universitárias. Rev. psiquiatr. clín. 2009; 36 (3): 83-88.

2. Morandé G, Celada J, Casas JJ. Prevalence of eating disorders in a Spanish school-age population. J. Adolesc. Health. 1999; 24(3): 212219.

3. Harrison K. Body image and eating disorders: definition and prevalence. Routledge Int. Handb. Child. Adolesc. Media. 2013; 224.

4. Trace SE, Baker JH, Peñas-Lledó E, Bulik CM. The Genetics of eating disorders. Annu. Rev. Clin. Psychol. 2013; 9(1): 589-620.

5. Thompson-Brenner H, Franko DL, Thompson DR, Grilo CM, Boisseau CL, Roehrig JP, et al. Race/ethnicity, education, and treatment parameters as moderators and predictors of outcome in binge eating disorder. J Consult Clin Psychol. 2013; 81 (4): 710-721.

6. Gaskin JL, Pulver AJ, Branch K, Kabore A, James T, Zhang J. Perception or reality of body weight: which matters to the depressive symptoms. J. Affect. Disord. 2013; 150(2): 350355.

7. Fandiño A, Giraldo SC, Martínez C, Aux CP, Espinosa R. Factores asociados con los trastornos de la conducta alimentaria en estudiantes universitarios en Cali, Colombia. Rev. Colomb. Médica. 2007; 38(4): 344-351.

8. Alvarenga $\mathrm{M}$ dos S, Lourenço BH, Philippi ST, Scagliusi FB. Disordered eating among Brazilian female college students. Cad. Saúde Pública. 2013; 29(5): 879-888.

9. Fernández ML, Otero MC, Castro YR, Prieto MF. Hábitos alimentarios e imagen corporal en 
estudiantes universitarios sin trastornos alimentarios. Int. J. Clin. Heal. Psychol. 2003; 3 (1): 23-33.

10. Fairburn CG, Harrison PJ. Eating disorders. The Lancet. 2003; 361(9355): 407-416.

11. Price $\mathrm{C}$, Morgan J. Introducing eating disorders. Medicine (Baltimore). 2012; 14 (1): 97-108.

12. American Psychiatric Association. Diagnostic and statistical manual of mental disorders: DSM-IVTR. Arlington, 2000.

13. Widiger TA, Samuel DB. Diagnostic categories or dimensions? A question for the diagnostic and statistical manual of mental disorders, fifth edition. J. Abnorm. Psychol. 2005; 114 (4): 494504.

14. Yager J. Weighty perspectives: contemporary challenges in obesity and eating disorders. Am. J. Psychiatry. 2000; 157 (6): 851-853.

15. Leal GV da S, Philippi ST, Polacow VO, Cordás TA, Alvarenga $\mathrm{M}$ dos $\mathrm{S}$. What is adolescents' eating disorder risk behavior? J. Bras. Psiquiatr. 2013; 62(1): 62-75.

16. Rodríguez A, Novalbos JP, Martinez JM, Ruiz MA, Fernandez JR, Jimenez D. Eating disorders and altered eating behaviors in adolescents of normal weight in a Spanish city. J. Adolesc. Health. 2001; 28 (4): 338-345.

17. Moya T, Fleitlich-Bilyk B, Goodman R. Brief report: Young people at risk for eating disorders in Southeast Brazil. J. Adolesc. 2006; 29(2): 313317.

18. Pinkston M, Martz D, Domer F, Curtin L, Bazzini $\mathrm{D}$, Smith L, et al. Psychological, nutritional, and energy expenditure differences in college females with anorexia nervosa vs. comparable-mass controls. Eat. Behav. 2001; 2(2): 169-181.

19. Pompili M, Mancinelli I, Girardi P, Ruberto A, Tatarelli R. Suicide in anorexia nervosa: A metaanalysis. Int. J. Eat. Disord. 2004; 36(1): 99-103.

20. Bodell LP, Joiner TE, Keel PK. Comorbidityindependent risk for suicidality increases with bulimia nervosa but not with anorexia nervosa. J. Psychiatr. Res. 2013; 47(5): 617-621.

21. Hoek HW, Van Hoeken D. Review of the prevalence and incidence of eating disorders. Int. J. Eat. Disord. 2003; 34(4): 383-396.

22. Cruzat C, Ramírez P, Melipillán R, Marzolo P. Trastornos alimentarios $y$ funcionamiento familiar percibido en una muestra de estudiantes secundarias de la comuna de Concepción, Chile. Psykhe Santiago. 2008; 17(1): 81-90.

23. Brownell KD. Handbook of eating disorders: physiology, psychology, and treatment of obesity, anorexia, and bulimia. Basic Books; 1986. 